\title{
The effect of the aging of liquid organic scintillators used for gamma-neutron separation
}

\author{
Jiř́ Janda ${ }^{1}$, Jaroslav Jánský ${ }^{2}$, Věra Mazánková ${ }^{2}$ František Cvachovec ${ }^{2}$ \\ ${ }^{1}$ University of Defence, NBC Defence Institute, Vyškov, Czech Republic \\ ${ }^{2}$ University of Defence, Faculty of Military Technology, Department of Mathematics and Physics, \\ Brno, Czech Republic \\ Corresponding author: jiri.janda@unob.cz
}

\begin{abstract}
Since the beginning of using liquid scintillators for gamma-neutron separation, there have been many articles dealing with long-term degradation especially due to oxygen presented during scintillator encapsulation. The effect of aging of liquid organic scintillators namely EJ301, EJ309 (both Eljen Technology), and new custom-made cocktails based on 1-Phenyl-3-(2,4,6-trimethylphenyl)-2pyrazoline and 2,5-Bis(5-tert-butyl-benzoxazol-2yl)thiophene fluors were investigated for more than half a year. The research was focused on the Compton edge shifting of gamma particles since the position is proportional to the light yield of the selected scintillator. Furthermore, the gamma-neutron separation was observed and quantified using FOM (Figure Of Merit) for samples prepared and filled under normal and nitrogen atmosphere during the mentioned period. All stated parameters of liquid scintillator NE213 manufactured by Nuclear Enterprises Limited opened more than three decades ago were measured and used for comparison.
\end{abstract}

Keywords - liquid organic scintillator; $\gamma$-n discrimination; long-term stability; scintillator aging.

\section{INTRODUCTION}

Q UENCHING due to dissolved oxygen is an important effect and must be avoided to obtain optimum scintillation efficiency. The explanation is given, that the quenching at aromatic molecules of the singlet state leads to the creation of a triplet state. The ground state of the oxygen molecule is a triplet and the next state is a singlet lying about $0.98 \mathrm{eV}$ over the ground state [1]. Therefore, oxygen molecules surrounding the aromatic molecules can absorb the energy of the singlet state of aromatic molecules and make a spin allowed transition to the triplet state causing a decrease of the fluorescence. Such a transition only occurs in aromatic molecules, which have an energy gap of S1-T1 greater than $0.98 \mathrm{eV}$ [2].

The magnitude of the quenching factor depends on the oxygen solubility in the solvent, which is determined by the external pressure and the temperature, and on the effect of oxygen quenching on the solvent and fluor and possibly other presented compounds (e.g. emulsifiers). At low concentrations, transfer quenching is predominant, but at increased concentrations, it decreases more rapidly than solute quenching. It appears likely that, at practical concentrations, near-ideal concentration for a given system, oxygen quenching of the fluor emission is the main factor.

Recently, few attempts were made to evaluate the basic performances of 18 years old scintillation cocktail. It has been shown that the 18-year storage has less impact on the basic performances (including pulse shape analysis) than batch-tobatch variability in cocktail compositions [3].

This study was focused on the evaluation of the long-term basic characteristics, such as Compton edge stability and g-n PSD, of the EJ301, EJ309, and different custom-made di-isopropylnaphthalene (DIPN) based liquid scintillation cocktails encapsulated under the nitrogen and air atmosphere.

\section{EXPERIMENTAL METHODS AND MATERIALS}

\section{A. Chemicals and materials}

The NE213 cocktail was obtained by Nuclear enterprises limited, UK (1986). Two commercially available scintillation cocktails were obtained by Eljen technology, USA - EJ301 scintillation cocktail based on xylene solution and 2,5Diphenyloxazole (PPO) and EJ309 scintillation cocktail based on DIPN solution and PPO. Two custom-made scintillators based on DIPN solution were prepared using 1-Phenyl-3-(2,4,6trimethyl-phenyl)-2-pyrazoline (labeled PYR) and 2,5-Bis(5tert-butyl-benzoxazol-2-yl)thiophene (labeled THIO) as luminophore. These luminophores were selected based on previous studies [4, 5]. Both custom-made cocktails were prepared in two concentrations of luminophore, 2 g.L $\mathrm{L}^{-1}$ (labeled 2) and 5 g.L $\mathrm{L}^{-1}$ (labeled 5), to verify the effect of quenching on luminophore concentration. Furthermore, two batches were prepared due to encapsulation under the nitrogen and ambient atmosphere.

Encapsulation of the liquid scintillators was performed using $20 \mathrm{~mL}$ glass vials with a low concentration of K-40 (Wheaton, UK) and the Teflon tape was used as a reflector.

An encapsulated stilbene crystal, with a diameter of $45 \mathrm{~mm}$ and a length of $45 \mathrm{~mm}$ is used for comparison.

The Cs-137 radionuclide was used for energy calibration in the same way as in [4]. The Cf-252 radionuclide was used to 
evaluate the neutron-gamma separation. The gamma-neutron discrimination was performed using NGA- 01 [6], which is the two-parameter spectrometric system and was used in earlier works $[4,7,8]$.

\section{B. Procedures}

\section{Preparation of scintillation cocktails}

The volume of $14 \mathrm{~mL}$ of the selected liquid scintillator was poured inside the $20 \mathrm{ml}$ glass vials with an upper lid containing a Teflon seal to ensure gas tightness and to better reflect photons to the photomultiplier tube (PMT). The glass vial was wrapped with Teflon tape to reflect photons. Two batches of previously mentioned scintillators were prepared this way; the first batch, labeled "N", was prepared under a nitrogen atmosphere and each liquid scintillator was bubbled with nitrogen for several minutes. After the selected period, the vial was properly closed. The second batch was prepared under an ambient atmosphere with no bubbling and was labeled as " $\mathrm{A}$ ". The batches during the whole observation period were stored in a dry, clean, and dark place with temperatures varying from $19^{\circ} \mathrm{C}$ to $24{ }^{\circ} \mathrm{C}$.

\section{Measurement setup}

The selected scintillator was placed on an RCA 8575 PMT and enclosed in a light-tight box. The radiation source was placed on the holder which was about $4 \mathrm{~cm}$ far from the scintillator horizontally. The PMT's anode output was connected to NGA-01. The high voltage of the PMT was set to $1650 \mathrm{~V}$ for each experiment. This should guarantee the fixed position of the Compton edge.

The Cs-137 was used for energy calibration, due to the creation of the Compton edge (CE) at $0.477 \mathrm{MeV}$. This energy belongs to a certain channel, which was determined using the derivative (differentiation) method. Due to the calibration, the term $\mathrm{MeV}$ electron equivalent (MeVee) could be introduced to quantify the light yield. The energy calibration was based on a linear function fit of Cs-137 measured data and zero energy channel and zero energy [4].

Gamma-neutron separation was measured using the same setup as was described in the paragraph dealing with the energy calibration, but instead of Cs-137, the neutron source Cf- 252 was used.

To guarantee satisfying results, more than a million pulses were acquired in each experiment. The count rate was in the range of from 150 up to $300 \mathrm{cps}$ for both mentioned radiation sources.

\section{RESULTS AND DISCUSSION}

The long-term observation of mentioned parameters started on the $30^{\text {th }}$ of September 2020 and ended on the $26^{\text {th }}$ of May 2021. Every month in this interval, the measurements of these two batches were performed using the same adjustment as described above.

\section{A. Light yield}

Since the magnitude of light collected at the photocathode of the PMT is proportional to the height of the electrical pulse, the light yield can be measured indirectly using the position of the Compton edge. As was stated in the introduction, the oxygen prevents excitation of the luminophore, thus causing quenching. Therefore, there should be some difference in the position of $\mathrm{CE}$ between samples prepared under the nitrogen and ambient atmosphere.

Since the diameter of the used PMT was $51 \mathrm{~mm}$, the variance in CE position of the used scintillators was performed. This variance of the $\mathrm{CE}$ was caused due to differently placed vials on the face of the PMT, different orientations of the vial, and different states of optical contact. It was observed, that the deviation was up to $7 \%$ for all scintillators based on 10 repetitions for each cocktail.

As can be seen in Table I, the differences in the position of the $\mathrm{CE}$ of samples prepared under nitrogen and ambient atmosphere on the first day corresponded with those previously published $[9,10]$, and the difference in quenching, i.e. position of $\mathrm{CE}$ between samples prepared under nitrogen/ambient atmosphere and lower and higher (optimal concentration $\mathrm{C}_{\mathrm{O}}$ ) is visible. Furthermore, the values of the standard deviation of the $\mathrm{CE}$ means of investigated scintillation cocktails and concentrations expressed in percentage are within the range of variation, when the samples are changed. The obtained data support previously published data, that the dissolved oxygen causes only quenching at some degree (based on the composition of the solvent-solute system), as was stated above. 
TABLE I

THE DEPENDENCE OF THE COMPTON EDGE POSITION ON THE DAY OF MEASUREMENT

\begin{tabular}{|c|c|c|c|c|c|c|c|c|c|c|c|c|}
\hline \multicolumn{13}{|c|}{ Compton edge position [channel] vs time [day of measurement] } \\
\hline Day & $\begin{array}{c}\text { EJ } 301 \\
\mathbf{N} \\
\end{array}$ & $\begin{array}{c}\text { EJ } 301 \\
\mathbf{A} \\
\end{array}$ & $\begin{array}{c}\text { EJ } 309 \\
\mathbf{N}\end{array}$ & $\begin{array}{c}\text { EJ } 309 \\
\mathbf{A}\end{array}$ & $\begin{array}{c}\text { PYR } 2 \\
\text { N }\end{array}$ & $\begin{array}{c}\text { PYR } 2 \\
\text { A }\end{array}$ & $\begin{array}{c}\text { PYR } 5 \\
\text { N }\end{array}$ & $\begin{array}{c}\text { PYR } 5 \\
\text { A }\end{array}$ & $\begin{array}{c}\text { THIO } \\
2 \mathrm{~N}\end{array}$ & $\begin{array}{c}\text { THIO } \\
2 \text { A }\end{array}$ & $\begin{array}{c}\text { THIO } \\
5 \mathrm{~N}\end{array}$ & $\begin{array}{c}\text { THIO } \\
5 \mathrm{~A}\end{array}$ \\
\hline 1. & 329 & 316 & 310 & 306 & 294 & 268 & 324 & 290 & 267 & 238 & 248 & 247 \\
\hline 9. & 298 & 260 & 323 & 316 & 291 & 283 & 307 & 297 & 254 & 252 & 260 & 255 \\
\hline 22. & 331 & 312 & 325 & 303 & 293 & 279 & 312 & 313 & 268 & 258 & 270 & 275 \\
\hline 36. & 287 & 277 & 315 & 316 & 296 & 286 & 317 & 308 & 266 & 255 & 251 & 258 \\
\hline 50. & 284 & 276 & 321 & 311 & 285 & 279 & 275 & 302 & 262 & 248 & 246 & 248 \\
\hline 66. & 294 & 292 & 310 & 300 & 280 & 286 & 281 & 305 & 263 & 247 & 253 & 252 \\
\hline 99. & 314 & 292 & 320 & 325 & 265 & 260 & 282 & 300 & 230 & 225 & 233 & 231 \\
\hline 126. & 280 & 286 & 319 & 303 & 276 & 261 & 264 & 293 & 262 & 243 & 239 & 249 \\
\hline 153. & 309 & 288 & 330 & 307 & 270 & 279 & 269 & 307 & 246 & 225 & 237 & 226 \\
\hline 188. & 304 & 284 & 331 & 314 & 248 & 269 & 266 & 299 & 259 & 229 & 231 & 228 \\
\hline 238. & 292 & 282 & 314 & 301 & 230 & 238 & 249 & 283 & 234 & 214 & 211 & 213 \\
\hline Mean & 302 & 288 & 322 & 309 & 275 & 272 & 286 & 300 & 255 & 240 & 244 & 244 \\
\hline$\sigma[\%]$ & 6 & 6 & 4 & 4 & 8 & 5 & 9 & 3 & 5 & 6 & 7 & 7 \\
\hline
\end{tabular}

HV of PMT was set to $1650 \mathrm{~V}$. N and A stand for nitrogen and air atmosphere respectively.

\section{B. Gamma-neutron discrimination}

In addition to measuring the stability of the $\mathrm{CE}$, the efficiency of gamma-neutron discrimination was also observed. To characterize the discrimination of two peaks originated from gamma and neutron radiation, the Figure of Merit (FOM) was used in the same way as in [4]. It was observed (Fig. 1) that the discrimination, especially at low energies, was improving with elapsing time. This improvement was noticeable for all cocktails in the energy range up to $0.4 \mathrm{MeVee}$. The highest improvement was observed in cocktails marked THIO. Since this luminophore is very problematic to dissolve in DIPN solvent, it is believed, that after the dissolution process, undissolved microscopic particles remained in the cocktail and they were gradually dissolving during the first weeks. On the other hand, the most stable cocktails were based on DIPN solvent and PPO and PYR luminophore prepared under a nitrogen atmosphere. The influence of the nitrogen atmosphere was especially noticeable for the xylene + PPO and DIPN + THIO systems. Systems composed of DIPN + PPO and DIPN + PYR did not show clear differences. 

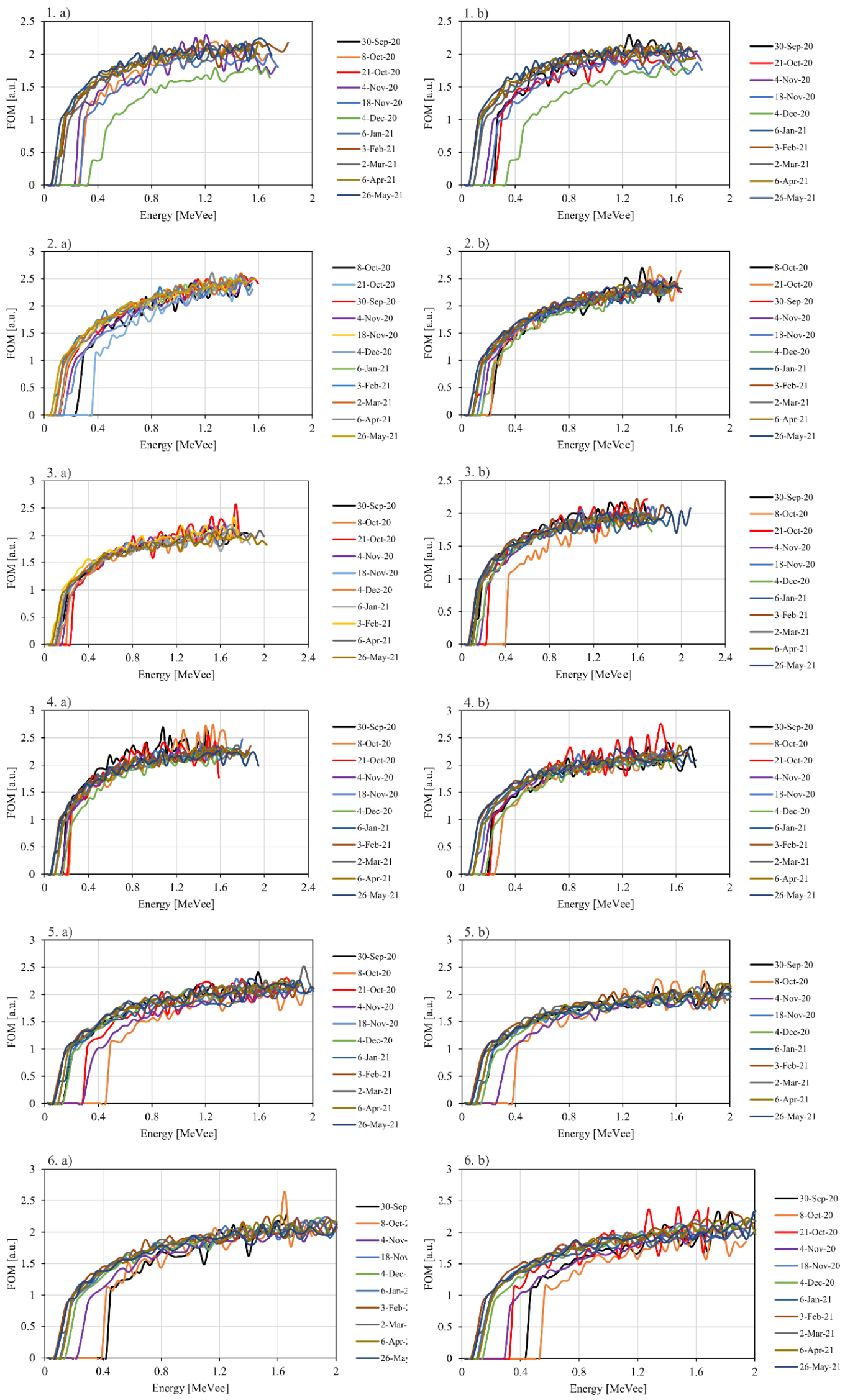

Fig. 1. The gamma-neutron separation of EJ301 (1), EJ309 (2), PYR 2 (3), PYR 5 (4), THIO 2 (5), and THIO 2 (6) scintillation cocktails prepared under nitrogen (a) and ambient (b) atmosphere expressed in FOM. 


\section{Comparison with NE213}

To verify and compare our results, the NE213 scintillator was measured. This scintillator has a similar composition as EJ301, which means it is based on xylene solvent with PPO as a luminophore. This bottle was opened in 1986 meaning that the effect of oxygen deterioration should be visible after more than
35 years.

To perform energy calibration, the Compton edge of Cs-137 had to be investigated. However, the data obtained when NE213 was measured was completely different and as can be seen in Fig. 2, the shape or even the evidence of CE was almost missing. The position of the CE was estimated to be at the $180^{\text {th }}$ channel at $\mathrm{HV}=1750 \mathrm{~V}$.
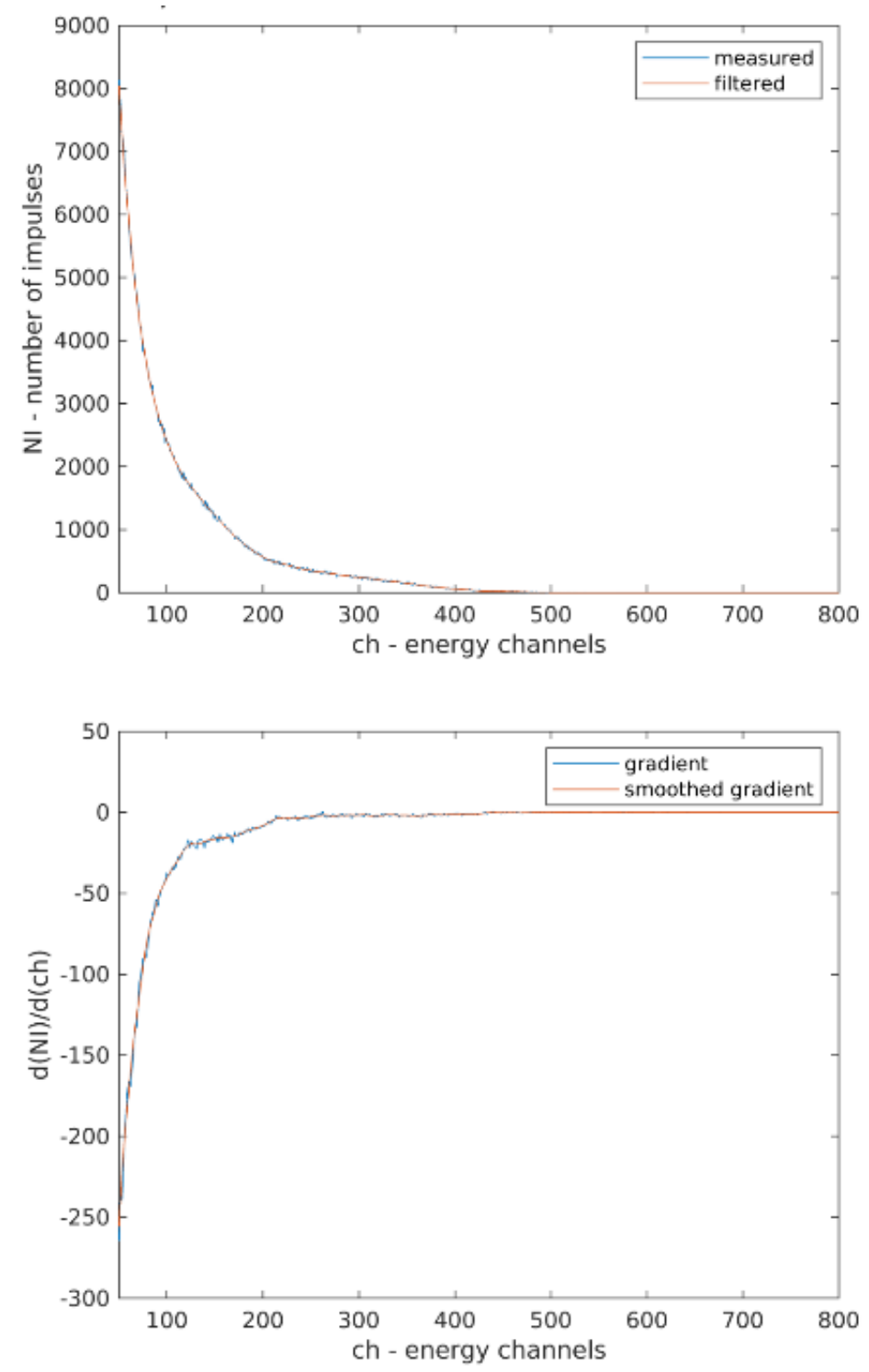

Fig. 2. Energy calibration using CE of Cs-137 at high voltage of PMT $1750 \mathrm{~V}$.

Despite the worse energy threshold for good discrimination, the gamma-neutron separation was still possible even after 35 years from the opening of the bottle and exposure to the cocktail to atmospheric oxygen. As can be seen (Fig. 3) in comparison with other scintillation cocktails (same geometry of measurement) the light output was lower and the energy resolution was bad. 

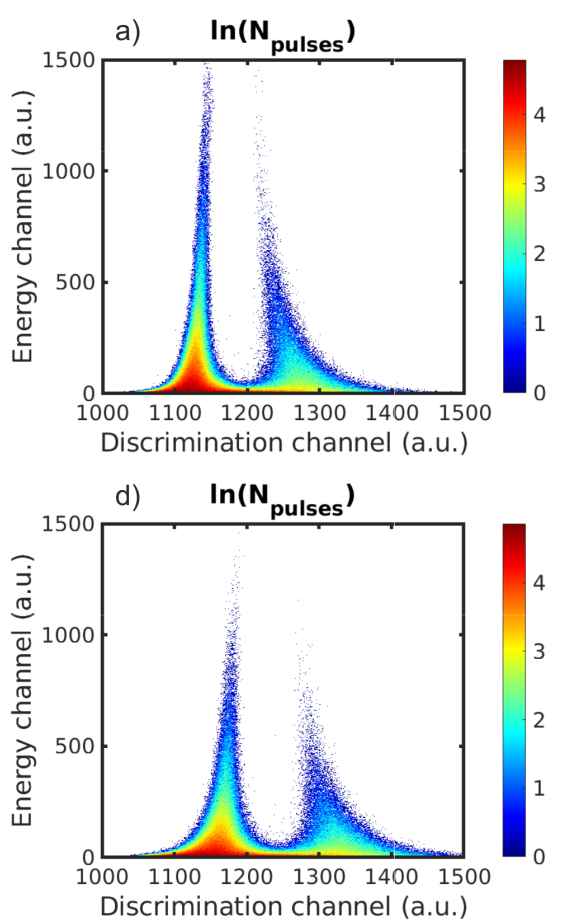
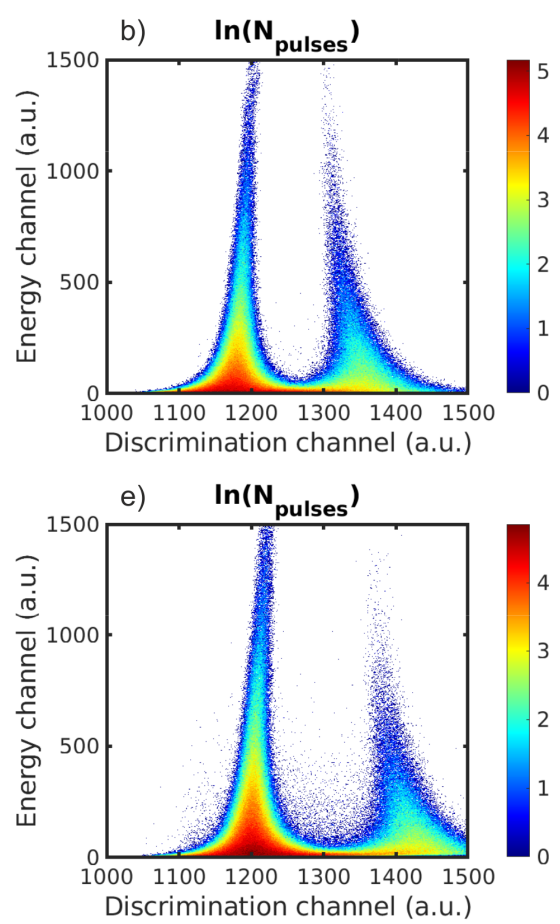
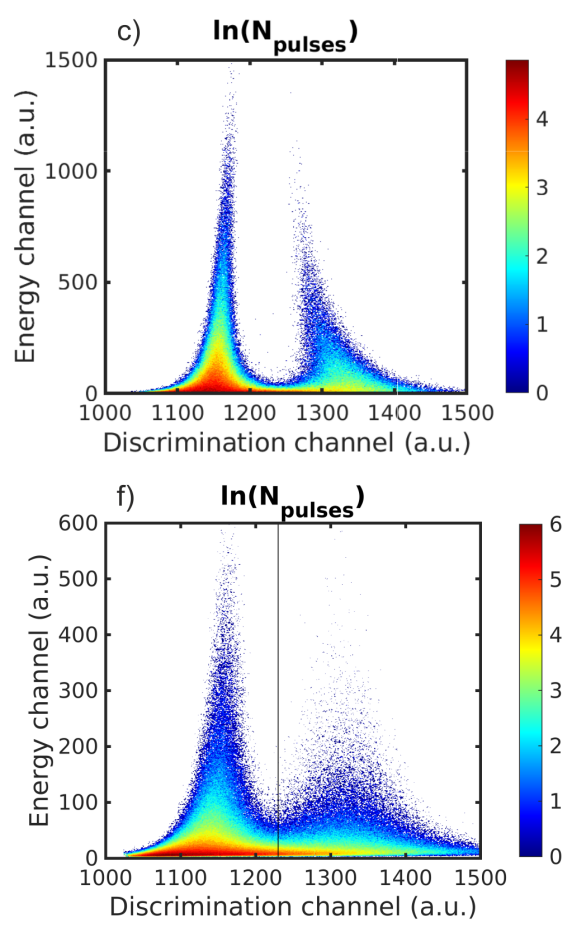

Fig. 3. Two-parameter histogram (left gammas, right neutrons) from the measurement of EJ301 (a), EJ309 (b), PYR 5 (c), THIO 5 (d), stilbene (e), and NE213 (f) using CE of Cs-137; HV of PMT in cases (a-e) was $1650 \mathrm{~V}$, in the case of (f) was $1750 \mathrm{~V}$.

\section{CONCLUSION}

This study aimed to evaluate the long-term performance of different scintillation cocktails prepared under nitrogen and ambient atmosphere. Especially, the focus was aimed to observe the stability of the Compton edge due to the light yield and, ability to discriminate between gamma rays and neutrons.

The effect of nitrogen and ambient atmosphere on CE position was following literature, where the higher shift in the position of the $\mathrm{CE}$ was observed at low concentration of luminophore and for higher concentration, the quenching was negligible in comparison of the variance in the position of $\mathrm{CE}$ due to sample changing, which was $7 \%$. It was proved, that the position of the $\mathrm{CE}$ in time is not stable with a negative slope at almost all cocktails meaning, that the light yield was constantly decreasing.

To compare observed results, the 35 years old and opened NE213 scintillation cocktail was measured and evaluated. It was demonstrated, that the cocktail didn't exhibit any visible Compton edge and therefore the energy calibration was very difficult to perform. Despite this difficulty, the cocktail was able to separate pulses originated from gamma rays and neutrons. The explanation of this worsening probably lies in chemical changes in the phosphor molecules caused by oxygen (e.g. oxidation) rather than in simple interrupting energy transfer from solvent to luminophore.

\section{ACKNOWLEDGMENT}

This research work has been carried out within the ADAR project (Accelerator Driven Advanced Reactor). The authors gratefully acknowledge the financial support from the Ministry of Education, Youth, and Sports of the Czech Republic under the INTER-ACTION research program (project No. LTAUSA 18198).

\section{REFERENCES}

[1] T. L. Brewer, "Oxygen quenching of the aromatic excited singlet state," J. Am. Chem. Soc., vol. 93, no. 3, Feb. 1971.

[2] X. Hua-Lin, D. Jing-Shan, and W. Nai-Yan, "Oxygen quenching in a LAB based liquid scintillator and the nitrogen bubbling model," Chinese Phys. C, vol. 34, no. 5, May 2010.

[3] X. gui Feng, Q. ge He, J. chen Wang, and J. Chen, "The long-term stability on basic performances of a diisopropylnaphthalene-based liquid scintillation cocktail," Appl. Radiat. Isot., vol. 70, no. 8, pp. 15361540, 2012.

[4] J. Jánský, J. Janda, V. Mazánková, and F. Cvachovec, "Optimization of composition of liquid organic scintillators for fast neutron spectrometry," Nucl. Instruments Methods Phys. Res. Sect. A Accel. Spectrometers, Detect. Assoc. Equip., p. 165523, 2021.

[5] J. Janda and E. Rajchl, "Study of new luminophores for use in modern scintillation cocktails," J. Lumin., vol. 201, pp. 390-396, Sep. 2018.

[6] Z. Matěj, J. Cvachovec, F. Cvachovec, and V. Prenosil, "Digitized two-parameter spectrometer for neutrongamma mixed field," Adv. Mil. Technol., vol. 7, pp. 51$58,2012$. 
[7] M. Koštál et al., "Comparison of fast neutron spectra in graphite and FLINA salt inserted in well-defined core assembled in LR-0 reactor," Ann. Nucl. Energy, vol. 83, pp. 216-225, Sep. 2015.

[8] M. Koštál et al., "Measurement and calculation of fast neutron and gamma spectra in well defined cores in LR-0 reactor," Appl. Radiat. Isot., vol. 120, pp. 45-50, Feb. 2017.

[9] J. B. Birks and F. W. K. Firk, "The Theory and Practice of Scintillation Counting," Phys. Today, vol. 18, no. 8, pp. 299-300, Aug. 1965.

[10] G. Laustriat and A. Coche, "Influence de la température sur les scintillateurs liquides," J. Phys. le Radium, vol. 21, no. 5, pp. 487-489, 1960. 